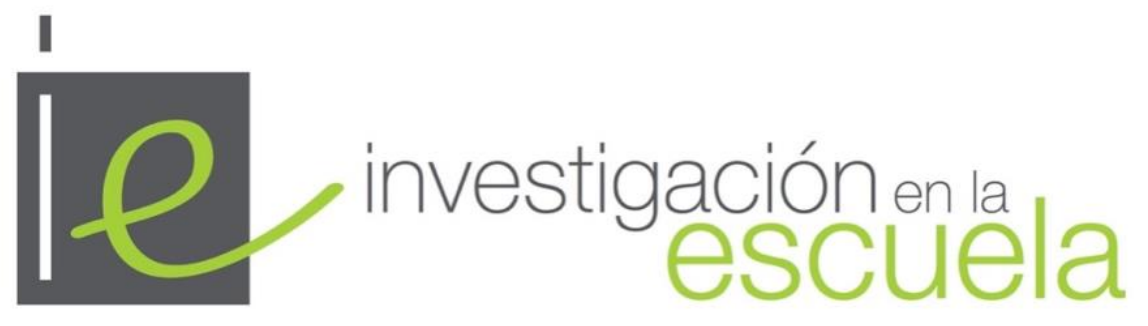

Revista de Investigación e Innovación Educativa nº 102, 2020 | e-ISSN 2443-9991

\title{
Aprendizaje Servicio. Análisis de una experiencia universitaria
}

\author{
Service Learning. Analysis of a university experience
}

Dr. David Herrera Pastor es Profesor Ayudante Doctor en la Facultad de Ciencias de la Educación y del Deporte de Melilla, Universidad de Granada (España)·dherrera@ugr.es · https://orcid.org/0000-0002-2198-5537

Cómo citar este artículo

Herrera Pastor, D. (2020). Aprendizaje Servicio. Análisis de una experiencia universitaria. Investigación en la Escuela, 102, 154-164. doi: http://doi.org/10.12795/IE.2020.1102.11

Resumen. En este artículo se revisan los fundamentos pedagógicos y sociales de una experiencia de Aprendizaje Servicio (ApS) llevada a cabo en la Universidad que resultó muy satisfactoria. En dicha experiencia se combinaron dos asignaturas (de dos Facultades distintas) y participaron 120 alumnos, 10 profesores y 12 entidades del tercer sector que trabajaban sobre situaciones de exclusión social. El objetivo era materializar un ApS a través del cual: 1) El alumnado trabajase en situaciones reales de esas entidades, para favorecer la inclusión de minorías (Diversidad funcional, Inmigración, LGTBI, etc.). 2) Realizase piezas audiovisuales con el propósito de sensibilizar y transformar el imaginario social al respecto. Dicha experiencia se examina a través de la vivencia concreta de uno de los grupos de alumnos (concretamente, el que tutorizó el autor de este artículo). Para escudriñar dicha experiencia se ha realizado un trabajo de campo que, además de otras acciones, fundamentalmente, se sustenta en entrevistas a representantes de todos los colectivos participantes en aquel proceso de aprendizaje: alumnado de las dos facultades, profesorado de las dos facultades, tutor y representante de la entidad colaboradora. Los datos evidencian las múltiples potencialidades educativas de este modelo de aprendizaje. Y los argumentos socio-pedagógicos que se esgrimen, muestran un proceso formativo muy adecuado, particularmente, para la enseñanza superior, y pretenden animar al profesorado universitario a su implementación.

\begin{abstract}
This article reviews the pedagogical and social foundations of a successful Service Learning experience at the University. The experience combined two subjects (from two different Faculties) and involved 120 students, 10 teachers and 12 third sector entities working on situations of social exclusion. The objective was to develop a Service Learning experience through which: 1) The students work in real situations of these entities, in order to include minorities (Disability, Immigration, LGTB+, etc.). And 2) producing audiovisual pieces with the purpose of transforming the general social conscience regarding these excluding realities. The Service Learning process is examined through the concrete experience of one of the groups of students (specifically, the one tutored by the author of this article). To examine this experience, fieldwork has been carried out which, in addition to other actions, is fundamentally based on interviews with representatives of all the members participating in the experience: students from the two faculties, teachers from the two faculties, tutor and a representative of the collaborating entity. Data show the multiple educational potentialities of this learning model. And the socio-pedagogical arguments put forward show a training process that is very appropriate, particularly for higher education, and aim to encourage university teachers to implement it.
\end{abstract}

\section{Palabras clave $\cdot$ Keywords}

Aprendizaje servicio, educación superior, educación social, comunicación, inclusión, transformación social. Service learning, higher education, social education, communication, inclusion, social transformation. 


\section{Introducción}

Este artículo emerge del trabajo llevado a cabo como consecuencia de un Proyecto de Innovación Educativa (PIE) que se implementó en la Universidad de Málaga utilizando la metodología Aprendizaje Servicio (ApS). Dicho trabajo se tradujo en una experiencia positiva para el alumnado y el profesorado, así como para un grupo de entidades y proyectos del tercer sector con las que se colaboró, que desempeñaban una labor socio-educativa con minorías y colectivos en riesgo de exclusión social.

Para materializar el proyecto de innovación se unieron dos asignaturas, una de la Facultad de Ciencias de la Educación y otra de la Facultad de Ciencias de la Comunicación de la mencionada universidad. El objetivo era realizar un proyecto conjunto, articulado a través de la metodología ApS, en el que el alumnado, organizado en grupos interdisciplinares (de ambas facultades), debía trabajar con las mencionadas entidades para contribuir en el desarrollo de su labor socio-educativa. La colaboración radicaba en elaborar varias piezas audiovisuales que les pudieran ayudar en ese sentido. En total participaron 120 alumnos (50\% de cada facultad) que se distribuyeron en 12 grupos (de 10 alumnos - 5 por cada facultad). Cada grupo trabajó con una entidad en particular.

En este escrito se van a revisar algunos de los principales fundamentos pedagógicos y sociales de la metodología, a través del análisis de la experiencia de aprendizaje servicio vivenciada por uno de esos grupos de alumnado, concretamente, el que realizó su colaboración con la Granja Escuela "El Pato" - FESE (Fundación Espacio Sin Exclusión). Una entidad que trabajaba por la inclusión (fundamentalmente, de personas con diversidad funcional) y por educar a la población respecto de la cultura de la diversidad.

Para examinar dicha experiencia se ha realizado el siguiente trabajo de campo: A) Entrevistas a representantes de los distintos colectivos participantes. En total 6 personas representativas de: el alumnado (de las dos facultades), la entidad del tercer sector colaboradora, los docentes responsables de las dos asignaturas (de las dos facultades) y el tutor que acompañó el proceso de este grupo concreto. B) Además, se recabaron las anotaciones que este último materializó en su cuaderno durante todo el proceso de tutorización. Y C) un registro audiovisual compuesto por los tres vídeos que realizó el grupo de alumnos en cuestión.

Los resultados evidencian las claves pedagógicas y sociales que posee la metodología ApS, que además de resultar innovadora, garantizando aprendizajes académicos y competenciales significativos que redundan positivamente en el desempeño profesional, está impregnada por una educación social, ética y política, particularmente, esperada de la población con estudios universitarios. Por su parte, el servicio que se lleva a cabo ayuda a una entidad social local a desarrollar una labor de educación social que contribuye a la mejora de la sociedad.

Las conclusiones sintetizan las ideas más destacadas que se desprenden del estudio, evidenciando las potencialidades de la metodología (ApS), para tratar de animar a que la docencia universitaria transite en esa dirección.

\section{El Aprendizaje Servicio como marco de referencia}

En este apartado se hace una revisión teórica concisa sobre la metodología Aprendizaje Servicio porque en el apartado Conclusiones se revisan, de manera sintética, algunos de los principales fundamentos pedagógicos y sociales que sustentan la metodología como modelo de aprendizaje. Los fundamentos que se revisan son los que emergen a partir del examen de la experiencia que se somete a estudio.

El Aprendizaje Servicio (ApS) es una pedagogía que se articula a través de un método didáctico que combina -en un proyecto socioeducativo planificado y materializado en consecuencia (Puig y Palos, 2006)-, formación reglada con servicio a la comunidad, incorporando sensibilización en causas sociales y acción política. Educación formal (Quintana Cábanas, 1993) y movilización social se unen para obtener un beneficio propio, añadiendo una enseñanza transversal relacionada con la justicia social y la ciudadanía.

En esencia, el ApS consiste en que los alumnos lleven a cabo un proyecto -un servicio- relacionado con las materias que están estudiando en una entidad real. Dicha entidad, que desempeña algún tipo de labor social, abre sus puertas a los aprendices para que estos participen de su trabajo. A través de esa colaboración ambas partes resultan favorecidas. Para los estudiantes supone una oportunidad de aprendizaje vivencial que posibilita el desarrollo de aprendizajes significativos (Ausubel, 1976) y relevantes (Marrero, 2007) en ese área de conocimiento y campo de acción, así como una formación competencial (conocimientos, actitudes, capacidades, etc.) (Batllé, 2011; Folgueiras y Martínez-Vivot, 2009) que permite su formación holística e integral. Ya que, además, todo ello se articula a través de un proceso donde la ética y la participación están implícitas (Stanton, 1990). Y para la entidad, porque recibe una contribución de nivel universitario de un 
grupo de colaboradores. El servicio responde a necesidades o demandas reales y, por tanto, resulta de utilidad. En ese sentido, aprendizaje y servicio mantienen una relación circular en la que ambas partes resultan beneficiadas: el aprendizaje adquiere sentido profesional, personal y cívico (Puig et al., 2011) y el servicio contribuye en la actividad de la entidad y, por ende, en la mejora de la comunidad. Para Tapia (2010), esta metodología permite a los discentes poner sus saberes al servicio de las necesidades de la sociedad, al tiempo que les permite aprender para el mundo académico, laboral, así como para la vida, en su plano individual y cívico.

\section{Metodología de investigación}

A través de los tres subapartados de este punto se dibujan los aspectos más significativos de la metodología de investigación utilizada. En el primero de ellos, se describe el escenario en el que tuvo lugar la experiencia sujeta a estudio, necesario para poder hacer una contextualización adecuada. En el segundo, se señala el foco de la investigación y se refieren las características básicas de la experiencia que se toma como objeto de estudio. Y, en el tercero, se comparten las acciones desarrolladas para realizar el trabajo de campo y se indica el proceso de categorización de los datos.

\subsection{Contexto: el Proyecto de Innovación}

El Proyecto de Innovación Educativa (PIE) fue aprobado y subvencionado por el servicio de Formación e Innovación de la Universidad de Málaga (código PIE: 15-156). En esencia, el proyecto de innovación consistía en implementar una metodología de aprendizaje innovadora que estuviese a la altura de las demandas que la formación universitaria en el marco del Espacio Europeo de Educación Superior planteaba en esos momentos. La metodología docente elegida era la metodología Aprendizaje Servicio (ApS), articulada de manera interdisciplinar para prestar un servicio que ayudase a las mencionadas entidades del tercer sector a implementar su labor principal y romper estereotipos existentes en el imaginario social contra las minorías o colectivos en situación de riesgo.

Para llevar a cabo el proyecto de innovación, se unieron dos asignaturas de dos Facultades distintas. Por un lado, estaba la asignatura: Infancia y Juventud en Riesgo Social, del Grado Educación Social (Facultad de Ciencias de la Educación), que contaba con 60 alumnos. Y, por otro lado, se encontraba la asignatura: Realización Audiovisual, del Grado Publicidad y Relaciones Públicas, de la Facultad de Ciencias de la Comunicación. Dicha asignatura también incorporaba 60 alumnos. En total, se unían 120 alumnos que se distribuyeron de manera equilibrada (Educación - Comunicación) en 12 grupos. Cada grupo inter-facultad contaba con 10 alumnos, cinco de la Facultad de Educación y cinco de la Facultad de Comunicación.

Se dividieron en 12 grupos porque 12 fueron las entidades y proyectos socio-educativos que nos habían contactado y demandado colaboración o se habían mostrado abiertos a recibir ese servicio. Las entidades participantes fueron: Cruz Roja Málaga; Cruz Roja Fuengirola; Mesa de trabajo comunitario "La Corta"; Asociación Trans; Casa Hogar "La Colonia"; Asociación de Vida Independiente "AVI"; Proyecto SEJ1366 "Trabajo en red y atención socioeducativa para la promoción de resiliencia en infancia en riesgo de exclusión social"; Grupo Resiliencia, Inclusión y Educación "RIEDU”; Casa Hogar "La buena vida"; Asociación Trencadís ROMI; Proyecto "Fronteras - Los Asperones"; y Granja Escuela "El Pato" - FESE (Fundación Espacio Sin Exclusión).

El servicio consistía en que cada grupo de alumnos se vinculaba a una de esas entidades o proyectos participantes y elaboraría tres piezas audiovisuales que habían de contribuir en su labor socio-pedagógica. La primera pieza audio-visual debía ser un "Teaser" (un producto más corto que un “Tráiler"), cuya duración había de ser inferior a 25 segundos, y debía servir para captar la atención de la audiencia en relación a la labor que dicha entidad estaba llevando a cabo. La segunda de las piezas audiovisuales era un "Spot", que no debía exceder los 45-50 segundos, cuya misión era, sintetizar la labor que realizaba la entidad, desde una perspectiva publicitaria. Y en tercer lugar se debía crear un Vídeo Corporativo, inferior a los cinco minutos, en el que, de manera más detenida, se explicase la labor que dicha entidad venía llevando a cabo.

Desde un punto de vista socio-educativo los objetivos de los audiovisuales eran: 1) luchar contra la exclusión que sufrían los usuarios de aquellas entidades y proyectos sociales, 2) promover su resiliencia y 3 ) hacer pedagogía social hacia el conjunto de la población, desmontando prejuicios y contribuyendo al desarrollo de una sociedad más justa e inclusiva. El alumnado de la Facultad de Educación era, fundamentalmente, el encargado de la parte pedagógica del servicio y el de Comunicación, de las cuestiones técnicas, de producción y de materialización de los audiovisuales. 
En el plano académico, aquel trabajo grupal (también individual, pues había tareas que tenían que realizar de manera singular) que se llevó a cabo durante un semestre, no solo daba cuerpo a la parte práctica de cada asignatura, sino que estaba plenamente vinculado con el contenido teórico de cada asignatura (el APRENDIZAJE). Y las piezas audiovisuales (el SERVICIO) resultaban una aportación para las entidades socio-educativas.

El ApS concluyó al final del semestre con una "Premiere" (estreno) de todas las piezas audiovisuales en un espacio específico para actividades culturales que tenía la Universidad. A dicha "Premiere" fueron invitados todos los participantes de la experiencia: Alumnado ( $\mathrm{y}$ familiares), Representantes de las entidades del tercer sector, Profesores de las asignaturas y Tutores de los grupos. Se organizó el encuentro para visionar juntos el producto de todo el trabajo realizado. El propósito era cerrar el círculo de la experiencia con un acto compartido en el que, además de aprender unos de otros (a través de los vídeos creados), el alumnado y las entidades sociales fueran reconocidos públicamente.

\subsection{Foco del estudio. El ApS con una entidad dedicada a la diversidad e inclusión}

Ese artículo sitúa su foco sobre los fundamentos pedagógicos y sociales de la metodología Aprendizaje Servicio llevada a cabo en la experiencia docente objeto de estudio. En concreto, en la experiencia protagonizada por uno de los grupos de alumnos integrante del Proyecto de Innovación descrito. ¿Por qué se pone el foco en la experiencia protagonizada por solo uno de los grupos? Porque fue el grupo que el autor de este artículo tutorizó directamente y haber tutorizado aquella experiencia le ubicaba en una posición muy propicia para desarrollar el estudio, pues conocía de primera mano todo el proceso.

Y es que para desarrollar el Proyecto de Innovación Educativa (PIE) diez docentes de las dos facultades mencionadas se unieron. La mayoría, no eran responsables de las asignaturas que vertebraban la innovación, eran docentes de la Universidad (de las facultades mencionadas más una docente de la Facultad de Psicología) que se incorporaron a la experiencia para colaborar en su desarrollo y aprender (haciendo) tutorizando algún grupo. Al autor de este artículo se le asignó el grupo que debía realizar el servicio en la Granja Escuela "El Pato" - FESE (Fundación Espacio Sin Exclusión). Dicha Granja Escuela desarrolla actividades educativas, generalmente, con alumnado de colegios (de infantil, primaria y secundaria), a través de las labores propias de la granja y de cuestiones relacionadas con el medio ambiente. En ella trabajaban personas funcionalmente diversas que en el ejercicio ordinario de la granja eran encargados de distintas tareas y, cuando recibían al alumnado de los colegios referidos, actuaban como monitores/educadores.

La Granja Escuela tenía dos objetivos principales, uno endógeno y otro exógeno. El primero de ellos era, como su propio acrónimo (FESE) indica, ser una fundación espacio sin exclusiones, es decir, conseguir, a través de su práctica de funcionamiento cotidiana crear, de manera fehaciente, ese lugar inclusivo en el que todos los participantes se pudieran desarrollar de manera plena. Lo que daba rienda a su objetivo exógeno, pues, en cuanto que evidenciaban desde la praxis que la consecución del primer objetivo era posible, real y sostenible, se convertían en un referente paradigmático que pretendía generar aprendizaje y conciencia en relación a la diversidad e inclusión. En definitiva, querían mostrar que verdaderamente se pueden crear espacios sociales inclusivos y, por tanto, hacer pedagogía social al respecto, educando al conjunto de la población para que rompan prejuicios y se avance hacia una sociedad más coherente en ese sentido.

A continuación, se comparten las tres piezas audiovisuales que realizó el grupo de alumnos (que protagonizó la experiencia ApS que se va a analizar) en colaboración con la Granja Escuela "El Pato" FESE (Fundación Espacio Sin Exclusión). Dichas piezas audiovisuales están colgadas en abierto en la plataforma digital YouTube:

. "Teaser": https://www.youtube.com/watch?v=nGUsefToG30

. "Spot": https://www.youtube.com/watch?v=AOroDcEZUms

- Vídeo Corporativo: https://www.youtube.com/watch?v=N1wXnOsrzDM

A través de ellas se puede apreciar de manera gráfica e ilustrada lo recién explicitado a partir de las narrativas audiovisuales elaboradas por aquel grupo.

\subsection{Trabajo de campo y categorización}

Con el propósito de conseguir sustrato empírico para examinar la experiencia se realizó trabajo de campo recurriendo a diversas fuentes de información y técnicas de recogida de datos. Dicho trabajo de campo, y la posterior categorización de los datos, se llevaron a cabo a partir de las tres categorías fundamentales que vertebran la metodología ApS: 1) el Aprendizaje; 2) la Dimensión social, ética y política que impregna el proceso; y 3) el Servicio. 
La principal estrategia de recolección de datos fue la entrevista. En particular, se entrevistó a un representante de cada uno de los colectivos involucrados en aquella experiencia. En concreto, se realizaron entrevistas a:

- Un representante del alumnado de la Facultad de Ciencias de la Educación.

- Un representante del alumnado de la Facultad de Ciencias de la Comunicación.

. El docente responsable de la asignatura de la Facultad de Ciencias de la Educación.

- El docente responsable de la asignatura de la Facultad de Ciencias de la Comunicación.

- Una persona con cargo de responsabilidad en la Granja Escuela "El Pato" - FESE (Fundación

Espacio Sin Exclusión).

- Y se contó con el testimonio del docente que tutorizó al grupo de alumnos en cuestión.

A cada uno de ellos se le inquirió en virtud del rol que había jugado durante el proceso. Todas las entrevistas fueron abiertas y semiestructuras y como objetivo tenían aprehender las interpretaciones de los distintos agentes respecto de la experiencia de Aprendizaje Servicio. Las diversas preguntas que se formularon giraron en torno a las tres categorías mencionadas.

Además de las entrevistas, se tuvo acceso a un registro documental: el cuaderno del tutor que había mentorizado a aquel grupo de alumnos a lo largo de todo el semestre. Las distintas entradas desarrolladas en el cuaderno mostraban la secuencia de acontecimientos vivenciado por dicho grupo de alumnos y se daba cuenta del proceso de trabajo seguido.

Por último, como registro audiovisual se tuvieron en cuenta las piezas audiovisuales realizadas por el grupo de alumnos. Los enlaces para acceder a dichos vídeos se encuentran en el subapartado 3.2 de este texto (como se indicó en el mencionado subapartado, dichos audiovisuales están publicados en internet en abierto). Esos vídeos son unas piezas gráficas que no solo ilustran el resultado del trabajo realizado por el grupo de alumnos, a través de ellos también se puede apreciar el proyecto en el que se enmarca la experiencia.

Como consecuencia de todo ello, se obtuvo un compendio de datos nutrido y rico que satisfacía las demandas indagatorias del trabajo de campo. Toda aquella información fue triangulada y estudiada de acuerdo al modelo de análisis de contenido (Herrera, 2016), en virtud de las tres categorías que vertebraban la metodología. Y a partir de la confrontación de los distintos testimonios recabados fueron aflorando distintos temas que a la postre fueron los que estructuraron los tres subapartados de los Resultados (uno por cada una de las categorías mencionadas) y, ulteriormente, las Conclusiones.

El proceso se llevó a cabo de manera inductiva. A continuación se inserta una tabla en la que se ilustran los principales temas emergidos en cada una de las categorías de análisis:

\section{Tabla 1}

Categorías de Análisis y Temas emergidos

\begin{tabular}{cl}
\hline Categoría & \multicolumn{1}{c}{ Temas Emergidos } \\
\hline Aprendizaje & $\begin{array}{l}\text { Experiencia de aprendizaje innovadora; Relación teoría-práctica/realidad; } \\
\text { Tipos de aprendizaje; Competencias; Ambiente de aprendizaje; } \\
\text { Motivación; Participación del alumnado. }\end{array}$ \\
$\begin{array}{c}\text { Dimensión social, ética y } \\
\text { política del proceso }\end{array}$ & $\begin{array}{l}\text { Formación interdisciplinar; Formación integral; Sensibilización en causas } \\
\text { sociales; Justicia Social; Bien común y Participación ciudadana. }\end{array}$ \\
Servicio & $\begin{array}{l}\text { Trabajo colaborativo; Solidaridad; Pedagogía Social y Cultura de la } \\
\text { Diversidad. }\end{array}$ \\
\hline
\end{tabular}

\section{Resultados}

A continuación, se interpretan y analizan los hallazgos obtenidos más destacados. Dicha interpretación y análisis se realiza en virtud de las tres categorías establecidas. En el primer subapartado, se abordan los temas emergidos en relación con el Aprendizaje. En el segundo, los vinculados con la categoría Dimensión social, ética y política del proceso. Y en el tercero, los que tienen que ver con el Servicio. 


\section{1. "Cuando tú sientes un aprendizaje se te queda para siempre"}

Un valor de la experiencia ApS es la conexión con la realidad. El alumnado apreciaba que aquella experiencia de aprendizaje se articulara en una entidad y en una situación real, superando las prácticas ficticias.

Yo sí recomendaría al profesorado universitario que utilizase el ApS, porque viene muy bien conocer gente de otras facultades, para aprender de otras formas de trabajo, tanto como hacer un trabajo en una entidad. Porque todo lo que hacíamos [en las otras asignaturas] solía ser ficticio, entonces llevar a cabo un proyecto real, pues la verdad que viene muy bien, ya que te pueden dar un feed-back... No es lo mismo que solo tengas la evaluación del profesor, en este caso teníamos el 'feed-back' de los compañeros de Educación como el de los de la Granja Escuela. (Alumna de la Facultad de Ciencias de la Comunicación).

El alumnado interpretó el servicio como una práctica que les permitía aprender desde la interacción y la experimentación. Por un lado, la configuración de grupos de trabajo interdisciplinares, de un equipo docente plural, así como la entidad social, fomentaba que el alumnado obtuviese retroalimentaciones diversas que cubrían distintas facetas, por lo que la formación resultaba poliédrica. Y, por otro lado, dicho servicio era una oportunidad para poner a prueba los conocimientos y aprendizajes que estaban interiorizando.

Aquella experiencia de aprendizaje estaba basada en el aprender haciendo, pero en el aprender haciendo con otros (...). Al final son aprendizajes muy vivenciales, son aprendizajes que no te los estudias en un libro, sino que los has vivido y que los has experimentado, que los has experimentado con personas concretas y en espacios concretos. Y esos aprendizajes al final se graban. (Profesor responsable de la asignatura de la Facultad de Ciencias de la Educación).

La entidad social fue un agente educativo activo que se involucró en el aprendizaje del alumnado. Lo hicieron porque era parte interesada, pero, sobre todo, porque la acción pedagógica era propia de su naturaleza. El alumnado de la Facultad de Educación estaba familiarizado con el discurso de la Granja Escuela "El Pato" - FESE, pero no así el de la Facultad de Comunicación.

El alumnado de Comunicación solo se mostraba preocupado por cuestiones técnicas, [pero] queríamos que entendieran cuáles eran nuestros principios. (Responsable de la entidad colaboradora).

El alumnado de Comunicación debía documentarse sobre el mensaje que se había de trasladar / el producto que debían "vender" (recuérdese que estaban estudiando la titulación / el Grado Publicidad y Relaciones Públicas), porque lo cierto es que desconocía la mayoría de aquellas ideas.

Para nada estábamos familiarizados con los conceptos de inclusión y diversidad que se querían trasladar. (Alumna de la Facultad de Ciencias de la Comunicación).

El trabajo del alumnado de la Facultad de Educación consistía en mediar en esa labor. Entre otras cosas, debían hacer un trabajo educativo con sus homólogos de la otra facultad, lo que resultaba un reto pedagógico. Debían manejar los conceptos que tenían que hacer comprender a sus colegas, que eran conceptos que estaban aprendiendo en su asignatura (exclusión, segregación, inclusión, resiliencia, educación, equidad, igualdad de oportunidades, discriminación positiva, etc.).

Nosotros hacíamos una labor más pedagógica y los de Comunicación ponían las herramientas (...). Para mí fue un reto muy grande tener que explicarles nuestra perspectiva de la educación y en nuestro caso con la Granja sobre la inclusión para que se plasmara en un vídeo... Al principio fue un choque brutal, pero luego se me quedaron sensaciones superbuenas (...), fue magnífico al final. (Alumna de la Facultad de Ciencias de la Educación).

La interacción entre los distintos agentes comprendía bastante dificultad (distintas culturas académicas, distintos enfoques, distintos valores, etc.), pero fue extraordinariamente rica, pues a través de ella se desarrollaron los procesos deseados y alcanzaron los resultados previstos.

He contactado con el grupo a través de una alumna de Educación Social y me comenta que existen problemas internos entre ellos y los de Comunicación. (Cuaderno del tutor del grupo: Entrada 5 Abril). [...] Parece que de la tutoría de hoy salen un poco más cohesionados. (Cuaderno del tutor del grupo: Entrada 25 Abril).

Al final los de Comunicación también consiguieron sentir lo que los de Educación queríamos explicarles. (Alumna de la Facultad de Ciencias de la Educación).

El resultado fue muy bueno, pero el proceso fue mejor. (Responsable de la entidad colaboradora).

El trabajo requerido a cada una de las partes y a cada uno de los alumnos en el proyecto colectivo común demandaba el desarrollo de competencias (trabajar en equipo, diseñar, organizar, coordinarse, etc.), pues no tenían solo que mostrar ciertos conocimientos, además debían conjugar, adecuadamente, elementos 
de muy distinta índole (actitudes, capacidades, emociones, valores, etc.) para completar el servicio de manera satisfactoria.

La experiencia suscitaba mogollón de competencias, después está el grado de adquisición de cada alumno, pero, en general, se adquirían un montón de competencias y de aprendizajes concretos. (Profesor responsable de la asignatura de la Facultad de Ciencias de la Educación).

Conforme el alumnado del grupo iba siendo consciente de la trascendencia que el trabajo que la Granja Escuela "El Pato" - FESE estaba desarrollando socialmente, se iban generando vínculos con el proyecto. Por eso, les resultaba gratificante formar parte de él:

Te sientes bien porque estás trabajando para ese tipo de entidad. (Alumna de la Facultad de Ciencias de la Comunicación).

Se dieron cuenta de que aquella realidad social (sobre diversidad e inclusión) necesitaba ser más trabajada socialmente. Implicarse de manera activa en aquel proyecto les llevaba a motivarse e involucrarse en la tarea en un grado mayor.

Yo soy partidaria de un aprendizaje que tú sientas, porque si no, no tiene mucha validez el aprendizaje. Por ejemplo, yo me imagino que los compañeros de Comunicación lo que aprendieron no se les va a olvidar como si te estudiaras un tema para un examen (...). Y para nosotras mismas, hubo conceptos y términos que aprendimos en ese caminar (...). Creo que te impregnas más del aprendizaje. Porque cuando tú sientes un aprendizaje, se te queda para siempre (...), se trata de un aprendizaje que te marca. Y creo que desde esta perspectiva y con el ApS se consigue más que desde otras perspectivas. (Alumna de la Facultad de Ciencias de la Educación).

Todo lo relacionado en este punto configuraba una experiencia de aprendizajes significativos y relevantes.

\subsection{Universidad y formación de ciudadanos críticos: "Toma de partido por el bien común"}

La Universidad, además de proporcionar una formación profesional especializada de nivel superior, debería contribuir en la formación de ciudadanos críticos.

... El sentido de la Universidad de crear ciudadanos críticos que no sean solamente superespecializados de sus áreas, pero que después de otras áreas no... (Profesor responsable de la asignatura de la Facultad de Ciencias de la Comunicación).

A través de aquella innovación, además de suscitar los aprendizajes académicos correspondientes, se pretendía estimular el desarrollo de la dimensión ciudadana de aquellos alumnos, a través del con-tacto ${ }^{1}$ con realidades / causas sociales de su entorno.

El compromiso que tenemos en la Universidad de formar a personas críticas relacionadas con nuestro entorno. (Profesor responsable de la asignatura de la Facultad de Ciencias de la Comunicación).

Causas sociales que, en ocasiones, resultan invisibles, pero que forman parte de la sociedad / de la comunidad circundantes, y en cuanto que afectan al bien común deben ser tratadas por el conjunto de la ciudadanía.

Articular el proceso formativo en torno a las vidas de personas en situación de riesgo, les hizo conocer y sentir una realidad para muchos desconocida que, además de desmontarles estereotipos y prejuicios al respecto, supuso un proceso educativo en ciudadanía activa.

El ApS en un sentido más amplio es educación política, es decir, eduquemos en ciudadanía, en valores sociales, en compromisos sociales. En ese sentido, hay una toma de partido por las cuestiones sociales, por las cuestiones públicas, en este caso sobre personas que están en situación desfavorecida. A mí no me cabe duda de que esa dimensión política estaba dentro del proyecto [vinculado] con el bien común, la comunidad. (Profesor responsable de la asignatura de la Facultad de Ciencias de la Educación).

Aprender a través de este tipo de asuntos, que persiguen la justicia social, intenta estimular el desarrollo de la ética y la toma de partido fundamentada, así como el empoderamiento del sujeto para que actúe en consecuencia. Lo que parece que debiera ser un propósito educativo ineludible para con todos los estudiantes, de manera particular con los universitarios.

\footnotetext{
${ }^{1}$ Con-tacto: Conocer con tacto, con sensibilidad.
} 


\subsection{Servicio, "un 'bimbazo' de romper muros"}

Del mismo modo que la Granja Escuela "El Pato" - FESE fue solidaria abriéndose a colaborar en aquella experiencia de aprendizaje, el servicio que recibió también fue un acto de generosidad hacia ellos del proyecto y, sobre todo, del alumnado, ya que de otro modo no habrían podido obtener aquellas piezas audiovisuales, pues no disponían de los recursos necesarios.

Llegan justos a los retos que tienen [en su día a día]. (Profesor responsable de la asignatura de la Facultad de Ciencias de la Comunicación).

Aquellos vídeos supusieron una aportación relevante para la entidad. Dicha entidad no tenía posibilidades de explotar su función exógena -de difusión de la cultura de la diversidad-, más allá de la labor que realizaban en la granja. Por lo que contribuyeron a satisfacer esa situación.

Nosotros no trabajábamos mucho el marketing en redes sociales, entonces era fantástico tener algo, de hecho, es de las pocas cosas que tenemos de la institución a nivel audiovisual. Es un buen servicio para nosotros, sin lugar a dudas. (Responsable de la entidad colaboradora).

A través de aquellas piezas audiovisuales se podía hacer llegar aquella cultura / aquella filosofía sobre la diversidad y la inclusión a muchas más personas y, por tanto, que su labor tuviera mayor alcance.

Tuvimos la capacidad de enseñarlo a muchas personas y que mediante imágenes las personas entendieran qué era por lo que se luchaba allí a diario. Entonces creo que fue un beneficio. La verdad es que fue un pepinazo porque conseguimos llegar a muchas más personas. (Alumna de la Facultad de Ciencias de la Educación).

Aquellos vídeos eran testimonio y prueba de lo que se estaba intentando trasladar. El formato audiovisual, unido al potencial y coherencia de la experiencia que se estaba compartiendo, posibilitó que aquellos mensajes tuviesen una mayor trascendencia desde un punto de vista pedagógico. Trascendencia que contribuía a transformar la cultura social y a mejorar el entorno desde una perspectiva inclusiva.

No es que te lo cuenten, es que lo ves. (Responsable de la entidad colaboradora).

Para mí fue brutal, porque se reflejó lo que era la realidad y rompió un montón de prejuicios (...). Vídeos así y experiencias así son los que rompen las barreras que tenemos con ciertos colectivos. Lo vi como un bimbazo de romper paredes y romper muros que de otra manera a lo mejor hubiese sido más complicado, porque por más que yo en mi entorno hable de la inclusión, si no lo ven no lo van a creer. Entonces fue una manera de: aquí lo tenéis. (Alumna de la Facultad de Ciencias de la Educación).

\section{Conclusiones}

A continuación, se presentan las ideas que se esgrimen a partir de los resultados obtenidos. Ideas que, de manera sintética, vienen a reforzar los principales fundamentos sociopedagógicos del Aprendizaje Servicio.

La formación que la sociedad actual demanda, en este caso en el ámbito de la enseñanza superior, debe alejarse de los postulados y tradiciones repetitivas y memorísticas que han predominado en la cultura académica a lo largo de los años. El ApS encarna una experiencia de aprendizaje innovadora que contribuye a una formación compleja y completa que mejora sustancialmente los procesos y resultados de aprendizaje de modelos anacrónicos, y se encuentra a la altura de los retos que la sociedad contemporánea demanda al sistema universitario (Francisco y Moliner, 2010).

El ApS es una pedagogía que posibilita el desarrollo de aprendizajes significativos y relevantes (Pérez Gómez et al, 2008). Eso quiere decir, que lo que se puede aprender a través de ella tiene verdadera trascendencia para el aprendiz, en este caso, no solo para su satisfactorio desenvolvimiento en el plano académico y profesional, sino también para el personal y social (Rodríguez-Gallego y Ordóñez-Sierra, 2015).

Como se ha visto en los resultados, para el alumnado el servicio es un escenario de aprendizaje situado (Díaz Barriga, 2003) -de los que Bernstein (1993) denomina secundarios-, en el que debe aplicar el conocimiento que está adquiriendo a través del plan de estudios. De ese modo, la teoría no se aprende separada de la práctica (Korthagen, 1999), por el contrario, el aprendiz se sumerge en una situación verdadera desafiante (Freire, 1971), para incorporar los conocimientos y competencias que corresponda con sentido (más otros muchos que emergen de manera colateral). 
El ApS no solo posibilita el desarrollo de conocimientos ${ }^{2}$ sobre una disciplina (de hecho, en la experiencia analizada hemos visto que se ha trabajado desde un enfoque interdisciplinar), además requiere de la conjugación de otras cualidades humanas -individuales, pero también sociales- que estimulan el desarrollo holístico, integral/interseccional (Konstantoni et al., 2017) y competencial del sujeto. A través de ella, también se pueden educar actitudes, capacidades, emociones, valores, criterios éticos, etc. (Brozmanovál et al, 2019).

El proceso formativo, por tanto, no se articula utilizando la imitación como vía de aprendizaje, sino la interacción (Luriia et al., 1979) y la experimentación (Piaget e Inhelder, 2000) como principales medios. En los resultados hemos visto cómo se articulaban ambos de manera paralela, pero también interrelacionada. Por supuesto, que se puede aprender de otros y mediante la observación, pero, desde el procesamiento individualizado de esa información, a través del conflicto cognitivo y la reflexión (Goldberg y Coufal, 2009), que han de llevar a una interiorización personalizada: la consolidación de esquemas cognitivos existentes o la creación de otros. En ese sentido, no es un modelo de aprendizaje que persiga la formación de profesionales técnicos, por el contrario, pretende el desarrollo de profesionales prácticos y críticos (Schön, 1992; Trillo Alonso, 1994).

Establecer como eje de la experiencia de aprendizaje una situación real como la analizada tiene varios propósitos (Gargallo et al, 2014; Herrera, 2018). Por un lado, busca que lo que se aprenda sea actual y, sobre todo, útil. Lo que contribuirá a que el alumnado sienta que lo que adquiere, además de no estar desfasado, tiene valía y resulta de provecho. Como se ha podido ver, que los discentes experimenten esa utilidad resulta gratificante y motivador, ya que aprecian cómo su trabajo (y por tanto todo lo que están aprendiendo) resulta fructífero (García García y Sánchez Calleja, 2017).

Que el servicio se llevase a cabo en entidades del tercer sector que trabajaban desarrollando acciones sociales, añadía un plus en ese sentido motivacional, ya que, de por sí, la tarea que realizaban apelaba a la solidaridad de los estudiantes y les movía a la acción. Lo que se traducía en participación activa, protagonismo e implicación (“engagement") (Boekaerts, 2016) en las labores a desarrollar. Además, el alumnado era consciente de que aquellas entidades no habrían podido obtener aquel servicio (aquellas piezas audio-visuales) de otro modo, lo que incentivaba su compromiso en la actividad. Todo aquello, les disponía muy favorablemente hacia la labor (servicio) a realizar y, en consecuencia, hacia el proceso de aprendizaje (Folgueiras et al., 2013).

Además de predisponer al alumnado de ese modo, la sensibilización sobre las causas sociales y la acción transformadora es un aprendizaje transversal idiosincrásico de esta metodología. Un aprendizaje que abunda sobre el pensamiento crítico y en la ciudadanía activa y responsable de los educandos (Martínez-Vivot y Folgueiras, 2015), tan necesaria para el desarrollo de las sociedades y, particularmente esperada del alumnado universitario.

Como se ha visto, las entidades sociales colaboradoras pretenden in-formar al conjunto de la población sobre el tipo de labor que realizan (Chiva et al., 2018). En la experiencia de aprendizaje estudiada, las piezas audiovisuales que comprendían el servicio tenían un propósito pedagógico de educación social. Pretendían formar a la sociedad en relación a las diversas causas sociales, para estimular la consecución de una práctica social más adecuada y coherente en ese sentido. En concreto, para la transformación de la comunidad en una sociedad más sensible, justa e inclusiva.

Se pone de manifiesto que esa labor de pedagogía social se lleva a cabo de manera más potente cuando se desarrolla de manera interdisciplinar. Los datos muestran que la relación establecida entre Educación y Comunicación fue muy adecuada y que la sinergia generada entre ambas posibilitaba una labor con mayor alcance que por separado. Lo que lleva a reivindicar mayor colaboración no solo entre dichas Ciencias, sino entre los distintos ámbitos del saber.

Todo lo relacionado hasta aquí abunda en la idea de que los procesos formativos se han de diseñar y ejecutar en cuanto que situaciones conectadas con la realidad que zambullan a los aprendices en procesos de aprendizaje significativos y relevantes estimulantes (Dewey, 1938). Albert Einstein, reflexionando sobre su faceta docente, formuló una frase que se hizo célebre: "Yo no enseño a mis alumnos, solo les proporciono las condiciones en las que puedan aprender". Procurar condiciones / situaciones para que el alumnado tenga la posibilidad de vivenciar experiencias de aprendizaje pedagógicamente potentes (Herrera et al., 2020) debe ser el reto de los sistemas educativos y, en particular, de los docentes (Rodríguez-Gallego, 2014). Reto que hay que afrontar, si verdaderamente se quiere tratar de proporcionar una formación de calidad (Tapia, 2014), en este caso en la etapa universitaria. Para ello, se han de conjugar todos (o la gran mayoría de) los elementos que se han desgranado en estas páginas. Y ello depende, fundamentalmente, de las universidades, pero,

\footnotetext{
2 Cono-cimientos: Conocer de manera cimentada/fundamentada. Pilares cognitivos con significado propio que estructuran el pensamiento y sobre los que se pueden construir otros esquemas mentales.
} 
sobre todo, de los docentes. De las primeras, porque deben apoyar, de un modo efectivo, el cambio de modelo de aprendizaje (de manera formativa, estructural, social, académica, etc.). Y de los segundos porque son el agente indispensable que ha de posibilitar su materialización.

\section{Referencias}

Ausubel, D. P. (1976). Psicología educativa: un punto de vista cognoscitivo. Trillas.

Batllé, R. (2011). ¿De qué hablamos cuando hablamos de aprendizaje-servicio? Crítica, 972, 49-54.

Bernstein, B. (1993). La estructura del discurso pedagógico. Morata.

Boekaerts, M. (2016). Engagement as an inherent aspect of the learning process. Learning and Instruction, 43, 76-83. Doi:10.1016/j.learninstruc.2016.02.001

Brozmanová, A., Heinzová, Z., Kurčikov, K., Nemcová, L. and Šolcová, J. (2019). Desarrollo de competencias claves a través del aprendizaje-servicio. Universidad, Escuela y Sociedad, 6, 34-54.

Chiva Bartoll, O., Capella Peris, C. y Martínez Usarralde, M. J. (2018). Aprendizaje-servicio y responsabilidad social universitaria: una apuesta por la mejora docente y el compromiso social. En O. Chiva y J. Gil (Coords.), Aprendizaje-servicio universitario: Modelos de intervención e investigación en la formación docente (pp. 5970). Octaedro.

Dewey, J. (1938). Experience and Education. Touchstone.

Díaz Barriga, F. (2003). Cognición situada y estrategias para el aprendizaje significativo. Revista Electrónica de Investigación Educativa, 5(2). http://redie.uabc.mx/redie/article/view/85/1396

Francisco, A. y Moliner, L. (2010). El aprendizaje servicio en la Universidad: una estrategia en la formación de ciudadanía crítica. Revista Electrónica Interuniversitaria de Formación del Profesorado, 13(4), 69-77.

Freire, P. (1971). Pedagogía del oprimido. Siglo veintiuno de España editores.

Folgueiras, P. y Martínez-Vivot, M. (2009). El desarrollo de competencias en la Universidad a través del Aprendizaje y Servicio Solidario. Revista Interamericana de Educación para la Democracia, 2(1), 55-76.

Folgueiras, P., Luna, E. y Puig, G. (2013). Aprendizaje y servicio: estudio del grado de satisfacción de estudiantes universitarios. Revista de Educación, 362, 159-185. doi:10.4438/1988-592X-RE-2011-362-157

García García, M. y Sánchez Calleja, L. (2017). El aprendizaje servicio y el desarrollo de las competencias emocionales en la formación inicial del profesorado. Contextos Educativos, 20, 127-145. http://doi.org/10.18172/con.2991

Gargallo, B., Morera, I., Iborra, S., Climent, M. J., Navalón, S. y García, E. (2014). Metodología centrada en el aprendizaje. Su impacto en las estrategias de aprendizaje y en el rendimiento académico de los estudiantes universitarios. Revista Española de Pedagogía, 259, 415-435.

Goldberg, L. R. y Coufal, K. L. (2009). Reflections on service-learning, critical thinking, and cultural competence. Journal of College Teaching \& Learning, 6(6), 39-50.

Herrera, D. (2016). Aportaciones metodológicas a la investigación biográfica, relacionadas con el procesamiento y la organización de los datos. Cuestiones Pedagógicas, 25, 145-160. DOI: http://dx.doi.org/10.12795/CP.2016.i25.11

Herrera, D. (2018). Investigación biográfica y educación inclusiva. Una innovación docente universitaria de enorme potencial. International Journal of Educational Research and Innovation, 10, 211-231.

Herrera, D., Padilla, M. T. y González-Monteagudo, J. (2020). Acompañamiento sociopedagógico, holismo y longitudinalidad: Claves de una buena práctica con un menor infractor. Revista Fuentes, 22(1), 53-64. DOI: 10.12795/revistafuentes.2020.v22.i1.05

Konstantoni, K., Kustatscher, M. y Emejulu, A. (2017). Travelling with intersectionality across time, place and space. Children's Geographies, 15, 1-5. https://doi.org/10.1080/14733285.2016.1255838

Korthagen, F. (1999). Linking theory and practice. Changing the pedagogy of teacher education. Educational researcher, 28, 4-17.

Luriia, A., Leontiev, A.N. y Vygotskii, L. S. (1979). Psicología y pedagogía. Akal

Martínez-Vivot, M. y Folgueiras Bertomeu, P. (2015). Evaluación participativa, Aprendizaje-Servicio y Universidad. Profesorado. Revista de curriculum y formación del profesorado, 19(1), 128-143. http://www.ugr.es/local/recfpro/rev191ART7.pdf

Marrero, J. (2007). Del aprendizaje significativo al aprendizaje relevante. Indivisa: Boletín de estudios e investigación, 8, 101-110.

Pérez Gómez, A. I., Soto Gómez, E., Serván Núñez, M. J. y Sola Fernández, M. (2008). Contextos y recursos para el aprendizaje relevante en la universidad. Junta de Andalucía y Akal.

Piaget, J. e Inhelder, B. (2000). The psychology of the child. Basic Books. 
Puig, J. M. y Palos, J. (2006). Rasgos pedagógicos del aprendizaje-servicio. Cuadernos de Pedagogía, 357, 6063.

Puig, J.M., Gijón, M., Martín, X. y Rubio, L. (2011). Aprendizaje servicio y Educación para la Ciudadanía. Revista de Educación, número extraordinario, 45-67.

Quintana Cábanas, J. M. (1993). Los ámbitos profesionales de la animación. Narcea.

Rodríguez-Gallego, M. (2014). El Aprendizaje-servicio como estrategia metodológica en la Universidad. Revista Complutense de Educación, 25(1), 95-113. https://doi.org/10.5209/rev_RCED.2014.v25.n1.41157

Rodríguez-Gallego, M. y Ordóñez-Sierra, R. (2015). Una experiencia de aprendizaje servicio en comunidades de aprendizaje. Profesorado. Revista de currículum y formación del profesorado, 19(1), 314-33. http://www.ugr.es/local/recfpro/rev191ART14.pdf

Schön, D. A. (1992). La formación de profesionales reflexivos: hacia un nuevo diseño de la enseñanza y el aprendizaje en las profesiones. Paidós.

Stanton, T. (1990). Service Learning: Grouping toward a definition. En J. Kendall (Ed.), Combining Service and Learning: A resource book for community and public service. Vol 1 (pp. 65-67). National Society for Internships and Experiential Education, Raleigh, NC.

Tapia, M. N. (2010). La propuesta pedagógica del 'aprendizaje-servicio': una perspectiva Latinoamericana. T₹̧boeCoen. Revista cientifica, 5, 23-44.

Tapia, M. N. (2014). La aportación del aprendizaje-servicio en el mundo. ¿De qué calidad educativa hablamos? Cuadernos de Pedagogía, 450, 54-56.

Trillo Alonso, F. (1994). El profesorado y el desarrollo curricular: tres estilos de hacer escuela. Cuadernos de Pedagogía, 228, 70-74. 